\title{
Low-Energy Constants from Resonance Chiral Theory
}

\author{
Antonio Pich \\ Departament de Física Teòrica, IFIC, Universitat de València-CSIC, \\ Apt. Correus 22085, E-46071 València, Spain \\ E-mail: Antonio.Pich@ific.uv.es
}

I discuss the recent attempts to build an effective chiral Lagrangian incorporating massive resonance states. A useful approximation scheme to organize the resonance Lagrangian is provided by the large- $N_{C}$ limit of QCD. Integrating out the resonance fields, one recovers the usual chiral perturbation theory Lagrangian with explicit values for the low-energy constants, parameterized in terms of resonance masses and couplings. The resonance chiral theory generates Green functions that interpolate between QCD and chiral perturbation theory. Analyzing these Green functions, both for large and small momenta, one gets QCD constraints on the resonance couplings and, therefore, information on the low-energy constants governing the Goldstone interactions.

8th Conference Quark Confinement and the Hadron Spectrum September 1-6 2008

Mainz, Germany 


\section{Chiral Symmetry}

With $n_{f}$ massless quark flavours, the QCD Lagrangian is invariant under global $S U\left(n_{f}\right)_{L} \otimes$ $S U\left(n_{f}\right)_{R}$ transformations of the left- and right-handed quarks in flavour space. The symmetry group spontaneously breaks down to the diagonal subgroup $S U\left(n_{f}\right)_{L+R}$ and $n_{f}^{2}-1$ pseudoscalar massless Goldstone bosons appear in the theory, which for $n_{f}=3$ can be identified with the eight lightest hadronic states $\phi^{a}=\{\pi, K, \eta\}$. These pseudoscalar fields are usually parameterized through the $3 \times 3$ unitary matrix $U(\phi)=u(\phi)^{2}=\exp \left\{i \lambda^{a} \phi^{a} / f\right\}$.

The Goldstone nature of the pseudoscalar mesons implies strong constraints on their interactions, which can be most easily analyzed on the basis of an effective Lagrangian containing only the Goldstone modes $[1,2,3]$. The low-energy effective Lagrangian is organized in terms of increasing powers of momenta (derivatives) and quark masses: $\mathscr{L}=\sum_{n} \mathscr{L}_{2 n}$. At lowest order, the most general effective Lagrangian consistent with chiral symmetry has the form [2]:

$$
\mathscr{L}_{2}=\frac{f^{2}}{4}\left\langle D_{\mu} U^{\dagger} D^{\mu} U+U^{\dagger} \chi+\chi^{\dagger} U\right\rangle, \quad \chi \equiv 2 B_{0}(s+i p)
$$

where $D_{\mu} U=\partial_{\mu} U-i r_{\mu} U+i U l_{\mu}$ and $\langle A\rangle$ denotes the flavour trace of the matrix $A$. The external Hermitian matrix-valued sources $l_{\mu}, r_{\mu}, s$ and $p$ are used to generate the corresponding left, right, scalar and pseudoscalar QCD Green functions and allow to incorporate the explicit breaking of chiral symmetry through the quark masses: $s=\mathscr{M}+\ldots, \mathscr{M}=\operatorname{diag}\left(m_{u}, m_{d}, m_{s}\right)$. The constants $B_{0}$ and $f$ are not fixed by symmetry requirements; one finds that $f$ equals the pion decay constant (at lowest order) $f=f_{\pi}=92.3 \mathrm{MeV}$, while $B_{0}$ is related to the quark condensate:

$$
B_{0}=-\frac{\langle\bar{q} q\rangle}{f^{2}}=\frac{M_{\pi}^{2}}{m_{u}+m_{d}}=\frac{M_{K^{0}}^{2}}{m_{s}+m_{d}}=\frac{M_{K^{ \pm}}^{2}}{m_{s}+m_{u}} .
$$

With only two low-energy constants, the lowest-order chiral Lagrangian $\mathscr{L}_{2}$ encodes in a very compact way all the Current Algebra results obtained in the sixties.

The symmetry constraints become less powerful at higher orders. At $O\left(p^{4}\right)$ we need ten additional coupling constants $L_{i}$ to determine the low-energy behaviour of the Green functions [2]:

$$
\mathscr{L}_{4}=L_{1}\left\langle D_{\mu} U^{\dagger} D^{\mu} U\right\rangle^{2}+L_{2}\left\langle D_{\mu} U^{\dagger} D_{v} U\right\rangle\left\langle D^{\mu} U^{\dagger} D^{v} U\right\rangle+\ldots
$$

One-loop graphs with the lowest-order Lagrangian $\mathscr{L}_{2}$ contribute also at $O\left(p^{4}\right)$. Their divergent parts are renormalized by the $\mathscr{L}_{4}$ couplings, which introduces a renormalization-scale dependence. The chiral loops generate non-polynomial contributions, with logarithms and threshold factors as required by unitarity, which are completely determined as functions of $f$ and the Goldstone masses.

The precision required in present phenomenological applications makes necessary to include corrections of $O\left(p^{6}\right)$ [4]. This involves contributions from $\mathscr{L}_{4}$ at one-loop and $\mathscr{L}_{2}$ at two-loops, which can be fully predicted [5]. However, the $O\left(p^{6}\right)$ chiral Lagrangian $\mathscr{L}_{6}$ contains 90 (23) independent local terms of even (odd) intrinsic parity $[5,6,7]$. The huge number of unknown couplings limits the achievable accuracy. Clearly, further progress will depend on our ability to estimate these chiral couplings, which encode the underlying QCD dynamics. 


\section{Resonance Chiral Theory}

The limit of an infinite number of quark colours is a very useful starting point to understand many features of QCD [8,9]. Assuming confinement, the strong dynamics at $N_{C} \rightarrow \infty$ is given by tree diagrams with infinite sums of hadron exchanges, which correspond to the tree approximation to some local effective Lagrangian. Hadronic loops generate corrections suppressed by factors of $1 / N_{C}$. At $N_{C} \rightarrow \infty$, QCD has a larger symmetry $U(3)_{L} \otimes U(3)_{R} \rightarrow U(3)_{L+R}$, and one needs to include in the matrix $U(\phi)$ a ninth Goldstone boson field, the $\eta_{1}$. Resonance chiral theory $(\mathrm{R} \chi \mathrm{T})$ $[10,11,12]$ provides an appropriate framework to incorporate the massive mesonic states [13].

Let us consider a chiral-invariant Lagrangian describing the couplings of resonance nonet multiplets $V_{i}^{\mu v}\left(1^{--}\right), A_{i}^{\mu v}\left(1^{++}\right), S_{i}\left(0^{++}\right)$and $P_{i}\left(0^{-+}\right)$to the Goldstone bosons. At lowest order in derivatives the interaction Lagrangian $\mathscr{L}_{R}$, linear in the resonance fields, takes the form [10]:

$$
\begin{aligned}
\mathscr{L}_{R}=\sum_{i} & \left\{\frac{F_{V_{i}}}{2 \sqrt{2}}\left\langle V_{i}^{\mu v} f_{+\mu v}\right\rangle+\frac{i G_{V_{i}}}{\sqrt{2}}\left\langle V_{i}^{\mu v} u_{\mu} u_{v}\right\rangle+\frac{F_{A_{i}}}{2 \sqrt{2}}\left\langle A_{i}^{\mu v} f_{-\mu v}\right\rangle\right. \\
& \left.+c_{d_{i}}\left\langle S_{i} u^{\mu} u_{\mu}\right\rangle+c_{m_{i}}\left\langle S_{i} \chi_{+}\right\rangle+i d_{m_{i}}\left\langle P_{i} \chi_{-}\right\rangle\right\},
\end{aligned}
$$

where $u_{\mu} \equiv i u^{\dagger} D_{\mu} U u^{\dagger}, f_{ \pm}^{\mu v} \equiv u F_{L}^{\mu v} u^{\dagger} \pm u^{\dagger} F_{R}^{\mu v} u$ with $F_{L, R}^{\mu v}$ the field-strength tensors of the $l^{\mu}$ and $r^{\mu}$ flavour fields and $\chi_{ \pm} \equiv u^{\dagger} \chi u^{\dagger} \pm u \chi^{\dagger} u$. The resonance couplings $F_{V_{i}}, G_{V_{i}}, F_{A_{i}}, c_{d_{i}}, c_{m_{i}}$ and $d_{m_{i}}$ are of $O\left(\sqrt{N_{C}}\right)$.

The lightest resonances have an important impact on the low-energy dynamics of the pseudoscalar bosons. Below the resonance mass scale, the singularity associated with the pole of a resonance propagator is replaced by the corresponding momentum expansion; therefore, the exchange of virtual resonances generates derivative Goldstone couplings proportional to powers of $1 / M_{R}^{2}$. At lowest order in derivatives, this gives the large $-N_{C}$ predictions for the $O\left(p^{4}\right)$ couplings of chiral perturbation theory $(\chi \mathrm{PT})[10]$ :

$$
\begin{aligned}
2 L_{1}=L_{2}=\sum_{i} \frac{G_{V_{i}}^{2}}{4 M_{V_{i}}^{2}}, & L_{3}=\sum_{i}\left\{-\frac{3 G_{V_{i}}^{2}}{4 M_{V_{i}}^{2}}+\frac{c_{d_{i}}^{2}}{2 M_{S_{i}}^{2}}\right\}, \quad L_{5}=\sum_{i} \frac{c_{d_{i}} c_{m_{i}}}{M_{S_{i}}^{2}}, \\
L_{8}=\sum_{i}\left\{\frac{c_{m_{i}}^{2}}{2 M_{S_{i}}^{2}}-\frac{d_{m_{i}}^{2}}{2 M_{P_{i}}^{2}}\right\}, & L_{9}=\sum_{i} \frac{F_{V_{i}} G_{V_{i}}}{2 M_{V_{i}}^{2}}, \quad L_{10}=\sum_{i}\left\{\frac{F_{A_{i}}^{2}}{4 M_{A_{i}}^{2}}-\frac{F_{V_{i}}^{2}}{4 M_{V_{i}}^{2}}\right\} .
\end{aligned}
$$

All these couplings are of $O\left(N_{C}\right)$, in agreement with the counting indicated in Table 1, while for the couplings of $O(1)$ we get: $2 L_{1}-L_{2}=L_{4}=L_{6}=L_{7}=0$.

Owing to the $U(1)_{A}$ anomaly, the $\eta_{1}$ field is massive and it is often integrated out from the low-energy chiral theory. In that case, the $S U(3)_{L} \otimes S U(3)_{R}$ chiral coupling $L_{7}$ gets a contribution from $\eta_{1}$ exchange $[2,10]$ :

$$
L_{7}=-\frac{f^{2}}{48 M_{\eta_{1}}^{2}}
$$

\section{Short-Distance Constraints}

The short-distance properties of the underlying QCD dynamics impose some constraints on the resonance parameters $[11,13]$. At leading order in $1 / N_{C}$, the two-Goldstone matrix element of 


\begin{tabular}{|c|r|c|c|c|}
\hline$i$ & \multicolumn{1}{|c|}{$L_{i}^{r}\left(M_{\rho}\right)$} & $O\left(N_{C}^{m}\right)$ & Source & $L_{i}^{N_{C} \rightarrow \infty}$ \\
\hline $2 L_{1}-L_{2}$ & $-0.6 \pm 0.6$ & $O(1)$ & $K_{e 4}, \pi \pi \rightarrow \pi \pi$ & 0 \\
$L_{2}$ & $1.4 \pm 0.3$ & $O\left(N_{C}\right)$ & $K_{e 4}, \pi \pi \rightarrow \pi \pi$ & 1.8 \\
$L_{3}$ & $-3.5 \pm 1.1$ & $O\left(N_{C}\right)$ & $K_{e 4}, \pi \pi \rightarrow \pi \pi$ & -4.3 \\
$L_{4}$ & $-0.3 \pm 0.5$ & $O(1)$ & Zweig rule & 0 \\
$L_{5}$ & $1.4 \pm 0.5$ & $O\left(N_{C}\right)$ & $F_{K}: F_{\pi}$ & 2.1 \\
$L_{6}$ & $-0.2 \pm 0.3$ & $O(1)$ & Zweig rule & 0 \\
$L_{7}$ & $-0.4 \pm 0.2$ & $O(1)$ & $\mathrm{GMO}, L_{5}, L_{8}$ & -0.3 \\
$L_{8}$ & $0.9 \pm 0.3$ & $O\left(N_{C}\right)$ & $M_{\phi}, L_{5}$ & 0.8 \\
$L_{9}$ & $6.9 \pm 0.7$ & $O\left(N_{C}\right)$ & $\left\langle r^{2}\right\rangle_{V}^{\pi}$ & 7.1 \\
$L_{10}$ & $-5.5 \pm 0.7$ & $O\left(N_{C}\right)$ & $\pi \rightarrow e v \gamma$ & -5.4 \\
\hline
\end{tabular}

Table 1: Phenomenological values $\left[O\left(p^{4}\right)\right]$ of the renormalized couplings $L_{i}^{r}\left(M_{\rho}\right)$ in units of $10^{-3}$. The large- $N_{C}$ predictions obtained within the single-resonance approximation are given in the last column.

the vector current is characterized by the vector form factor

$$
F_{V}(t)=1+\sum_{i} \frac{F_{V_{i}} G_{V_{i}}}{f^{2}} \frac{t}{M_{V_{i}}^{2}-t} .
$$

Since $F_{V}(t)$ should vanish at infinite momentum transfer $t$, the resonance couplings should satisfy

$$
\sum_{i} F_{V_{i}} G_{V_{i}}=f^{2}
$$

Similarly, the matrix element of the axial current between one Goldstone and one photon is parameterized by the so-called axial form factor $G_{A}(t)$, which vanishes at $t \rightarrow \infty$ provided that

$$
\sum_{i}\left(2 F_{V_{i}} G_{V_{i}}-F_{V_{i}}^{2}\right) / M_{V_{i}}^{2}=0 .
$$

Requiring the scalar form factor $F^{S}(t)$, which governs the two-pseudoscalar matrix element of the scalar quark current, to vanish at $t \rightarrow \infty$, one gets the constraints [14]:

$$
4 \sum_{i} c_{d_{i}} c_{m_{i}}=f^{2}, \quad \quad \sum_{i} c_{m_{i}}\left(c_{m_{i}}-c_{d_{i}}\right) / M_{S_{i}}^{2}=0 .
$$

Since gluonic interactions preserve chirality, the two-point function built from a left-handed and a right-handed vector quark currents $\Pi_{L R}(t)$ satisfies an unsubtracted dispersion relation. In the chiral limit, it vanishes faster than $1 / t^{2}$ when $t \rightarrow \infty$; this implies the well-known Weinberg conditions [15]:

$$
\sum_{i}\left(F_{V_{i}}^{2}-F_{A_{i}}^{2}\right)=f^{2}, \quad \sum_{i}\left(M_{V_{i}}^{2} F_{V_{i}}^{2}-M_{A_{i}}^{2} F_{A_{i}}^{2}\right)=0 .
$$

The two-point correlators of two scalar or two pseudoscalar currents would be equal if chirality was preserved. For massless quarks, $\Pi_{S S-P P}(t)$ vanishes as $1 / t^{2}$ when $t \rightarrow \infty$, with a coefficient proportional to $\alpha_{s}\langle\bar{q} \Gamma q \bar{q} \Gamma q\rangle \sim \alpha_{s}\langle\bar{q} q\rangle^{2} \sim \alpha_{s} B_{0}^{2}$. Imposing this behaviour, one gets [16]:

$$
8 \sum_{i}\left(c_{m_{i}}^{2}-d_{m_{i}}^{2}\right)=f^{2}, \quad \sum_{i}\left(c_{m_{i}}^{2} M_{S_{i}}^{2}-d_{m_{i}}^{2} M_{P_{i}}^{2}\right)=3 \pi \alpha_{s} f^{4} / 4
$$




\section{Single-Resonance Approximation}

Let us approximate each infinite resonance sum with the first meson-nonet contribution. This is meaningful at low energies where the contributions from higher-mass states are suppressed by their corresponding propagators. The resulting short-distance constraints are matching conditions between an effective theory below the scale of the second resonance multiplets and the underlying QCD dynamics. With this approximation, Eqs. (3.2), (3.3) and (3.5) determine the vector and axial-vector couplings in terms of $M_{V}$ and $f$ [11]:

$$
F_{V}=2 G_{V}=\sqrt{2} F_{A}=\sqrt{2} f, \quad M_{A}=\sqrt{2} M_{V} .
$$

The scalar [14] and pseudoscalar parameters are obtained from (3.4) and (3.6) [13]:

$$
c_{m}=c_{d}=\sqrt{2} d_{m}=f / 2, \quad M_{P}=\sqrt{2} M_{S}(1-\delta)^{1 / 2} .
$$

The last relation involves a small correction $\delta \approx 3 \pi \alpha_{s} f^{2} / M_{S}^{2} \sim 0.08 \alpha_{s}$, which we can neglect together with the tiny effects from light quark masses.

Inserting these predictions into Eqs. (2.2), one finally gets all $O\left(p^{4} N_{C}\right) \chi \mathrm{PT}$ couplings, in terms of $M_{V}, M_{S}$ and $f$ :

$$
\begin{gathered}
2 L_{1}=L_{2}=\frac{1}{4} L_{9}=-\frac{1}{3} L_{10}=\frac{f^{2}}{8 M_{V}^{2}}, \\
L_{3}=-\frac{3 f^{2}}{8 M_{V}^{2}}+\frac{f^{2}}{8 M_{S}^{2}}, \quad L_{5}=\frac{f^{2}}{4 M_{S}^{2}}, \quad L_{8}=\frac{3 f^{2}}{32 M_{S}^{2}} .
\end{gathered}
$$

The last column in Table 1 shows the results obtained with $M_{V}=0.77 \mathrm{GeV}, M_{S}=1.0 \mathrm{GeV}$ and $f=92 \mathrm{MeV}$. Also shown is the $L_{7}$ prediction in (2.3), taking $M_{\eta_{1}}=0.80 \mathrm{GeV}$. The agreement with the measured values is a clear success of the large $-N_{C}$ approximation. It demonstrates that the lightest resonance multiplets give indeed the dominant contributions at low energies.

Corrections induced by $\mathrm{R} \chi \mathrm{T}$ couplings quadratic in the resonance fields have been considered $[17,18]$. Although they slightly modify some of the previous relations, the general pattern remains so that all $O\left(p^{4} N_{C}\right) \chi \mathrm{PT}$ couplings are still successfully determined in terms of resonance masses and the pion decay constant. The possible effect of more exotic $2^{++}$and $1^{+-}$resonance exchanges has been analyzed recently. The short-distance constraints eliminate any possible contribution to the $L_{i}$ couplings from $1^{+-}$exchange and only allow a tiny $2^{++}$contribution to $L_{3}, L_{3}^{T}=0.16 \cdot 10^{-3}$, which is negligible compared to the sum of vector and scalar contributions [19]. This small tensor contribution had been previously obtained in the SU(2) theory [20].

The study of other Green functions provides further matching conditions between the hadronic and fundamental QCD descriptions. Clearly, it is not possible to satisfy all of them within the single-resonance approximation, since QCD requires an infinite number of massive states. A useful generalization is the so-called Minimal Hadronic Ansatz, which keeps the minimum number of resonances compatible with all known short-distance constraints for the problem at hand [21].

\section{Determination of $\mathbf{O}\left(\mathbf{p}^{6}\right)$ Low-Energy Couplings}

The most general $\mathrm{R} \chi \mathrm{T}$ Lagrangian contributing to the $O\left(p^{6}\right) \chi \mathrm{PT}$ couplings has been recently constructed in Ref. [12]. A priori the Lagrangian contains a long list of possible operators, including terms with one $\left[O\left(p^{4}\right)\right]$, two $\left[O\left(p^{2}\right)\right]$ and three $\left[O\left(p^{0}\right)\right]$ resonance fields. Many of them can 
be eliminated, using the equations of motion, field redefinitions and algebraic identities. The functional integration of the resonance fields has been completed, obtaining the large- $N_{C}$ resonance contributions to all $O\left(p^{6}\right) \chi \mathrm{PT}$ couplings $C_{i}$ in terms of resonance parameters. Those low-energy constants which don't get any resonance contribution have been identified and useful relations among different couplings have been obtained. However, there remain still many unknown resonance parameters which require a further investigation of short-distance QCD constraints. A complete matching between $\mathrm{QCD}$ and $\mathrm{R} \chi \mathrm{T}$ has not yet been achieved at this order.

Some $O\left(p^{6}\right) \chi \mathrm{PT}$ couplings have been already determined by studying an appropriate set of three-point functions [22, 23, 24, 25, 26, 27]. For instance, the analysis of the $\langle V A P\rangle$ Green function allows to derive the values $[12,25]$ :

$$
\begin{aligned}
C_{78} & =\frac{f^{2}\left(3 M_{A}^{2}+4 M_{V}^{2}\right)}{8 M_{V}^{4} M_{A}^{2}}-\frac{f^{2}}{16 M_{V}^{2} M_{P}^{2}}, & C_{82} & =-\frac{f^{2}\left(4 M_{A}^{2}+5 M_{V}^{2}\right)}{32 M_{V}^{4} M_{A}^{2}}-\frac{f^{2}}{32 M_{A}^{2} M_{P}^{2}}, \\
C_{87} & =\frac{f^{2}\left(M_{A}^{4}+M_{V}^{4}+M_{A}^{2} M_{V}^{2}\right)}{8 M_{V}^{4} M_{A}^{4}}, & C_{88} & =-\frac{f^{2}}{4 M_{V}^{4}}+\frac{f^{2}}{8 M_{V}^{2} M_{P}^{2}}, \\
C_{89} & =\frac{f^{2}\left(3 M_{A}^{2}+2 M_{V}^{2}\right)}{4 M_{V}^{4} M_{A}^{2}}, & C_{90} & =\frac{f^{2}}{8 M_{V}^{2} M_{P}^{2}} .
\end{aligned}
$$

From a similar analysis of the $\langle S P P\rangle$ Green function, one obtains [12, 26]:

$$
C_{12}=-\frac{f^{2}}{8 M_{S}^{4}}, \quad C_{34}=\frac{3 f^{2}}{16 M_{S}^{4}}+\frac{f^{2}}{16}\left(\frac{1}{M_{S}^{2}}-\frac{1}{M_{P}^{2}}\right)^{2}, \quad C_{38}=\frac{f^{2}}{8 M_{S}^{4}}-\frac{f^{2}}{16 M_{P}^{4}} .
$$

The couplings $C_{12}$ and $C_{34}$ govern the amount of SU(3) breaking in the $K_{l 3}$ form factor at zero momentum transfer and, therefore, have important implications in the determination of $\left|V_{u s}\right|$ [26].

\section{Subleading $1 / \mathbf{N}_{\mathbf{C}}$ Corrections}

The large $-N_{C}$ limit provides a very successful description of the low-energy dynamics [13]. However, we are still lacking a systematic procedure to incorporate contributions of next-to-leading order (NLO) in the $1 / N_{C}$ counting. The first efforts concentrated in pinning down the most relevant subleading effects, such as the resonance widths which regulate the corresponding poles in the meson propagators [28], or the role of final state interactions in the physical amplitudes [14, 28, $29,30]$.

More recently, methods to determine the low-energy constants of $\chi \mathrm{PT}$ at the next-to-leading order in $1 / N_{C}$ have been developed $[17,18,31,32]$. This is an important issue because the dependence of the $\chi \mathrm{PT}$ couplings with the renormalization scale is a subleading effect in the $1 / N_{C}$ counting. Since the usual resonance-saturation estimates have been performed at $N_{C} \rightarrow \infty$, they are unable to control the renormalization-scale dependence of the low-energy couplings (at which value of $\mu$ the estimates apply?).

Quantum loops including virtual resonance propagators constitute a major technical challenge. Their ultraviolet divergences require higher-dimensional counterterms, which could generate a problematic behaviour at large momenta [32]. Thus, it is necessary to investigate the short-distance QCD constraints at the next-to-leading order in $1 / N_{C}$. A first step in this direction was achieved 
through a one-loop calculation of the vector form factor in the $\mathrm{R} \chi \mathrm{T}$ [32], which demonstrated that the matching with the underlying QCD dynamics strongly constrains the ultraviolet behaviour of $\mathrm{R} \chi \mathrm{T}$, determining the renormalized couplings needed for this particular calculation. This fact appears to be quite general [33] and has been further corroborated through a recent investigation of the full one-loop generating functional that arises from $\mathrm{R} \chi \mathrm{T}$ with only one multiplet of scalar and pseudoscalar resonances [34].

Using analyticity and unitarity, it is possible to avoid all technicalities associated with the renormalization procedure, reducing the calculation of one-loop Green functions to tree-level diagrams plus dispersion relations $[17,18]$. This allows to understand the underlying physics in a much more transparent way. In particular, the subtle cancellations among many unknown renormalized couplings found in [32] and the relative simplicity of the final result can be better understood in terms of the imposed short-distance constraints.

As an example, let us consider the difference between the vector and axial-vector two-point functions $\Pi_{V-A}(t) \equiv \Pi_{V V}(t)-\Pi_{A A}(t)$. Its low-energy behaviour is dictated by $\chi \mathrm{PT}[2,5,35]$ :

$\Pi_{V-A}(t)=\frac{2 f^{2}}{t}-8 L_{10}^{r}(\mu)-\frac{\Gamma_{10}}{4 \pi^{2}}\left(\frac{5}{3}-\ln \frac{-t}{\mu^{2}}\right)+t\left[16 C_{87}^{r}(\mu)-\frac{\Gamma_{87}^{(L)}}{2 \pi^{2} f^{2}}\left(\frac{5}{3}-\ln \frac{-t}{\mu^{2}}\right)\right]+O\left(N_{C}^{0} t\right)$,

with $\Gamma_{10}=-1 / 4$ and $\Gamma_{87}^{(L)}=-L_{9}^{r}(\mu) / 2$. The couplings $f^{2}, L_{10}$ and $C_{87}$ are of $O\left(N_{C}\right)$, while $\Gamma_{10}$ and $\Gamma_{87}^{(L)} / f^{2}$ are of $O\left(N_{C}^{0}\right)$ and represent a NLO effect. The term $2 f^{2} / t$ contains the pole generated by the Goldstone-boson exchange. In the large $-N_{C}$ limit, $\Pi_{V-A}(t)$ receives in addition tree-level contributions from vector and axial-vector exchanges, which are easily computed within $\mathrm{R} \chi \mathrm{T}$. Expanding the $\mathrm{R} \chi \mathrm{T}$ expression in powers of momenta, one recovers the resonance-exchange predictions for the low-energy couplings $L_{10}$ and $C_{87}$ in Eqs. (4.3) and (5.1).

At NLO in $1 / N_{C}, \Pi_{V-A}(t)$ contains one-loop contributions from two-body exchanges of Goldstone bosons and heavy resonances, which give rise to ultraviolet divergences. However, these loop corrections can be fully determined from their finite absorptive contributions, through dispersive relations. The ultraviolet behaviour is then parameterized through the corresponding subtraction constants, which are fixed by the short-distance QCD behaviour requiring the correlator to vanish faster than $1 / t^{2}$ at infinite momentum. The contributions from the dominant $\pi \pi, \pi V, \pi A$, $\pi S$ and $\pi P$ exchanges have been computed in Ref. [18]. It is remarkable that, imposing a good short-distance behaviour for the corresponding vector and axial-vector spectral functions, one fully determines the relevant contributing form factors within the single resonance approximation. The low momentum expansion of the resulting $\Pi_{V-A}(t)$ correlator reproduces Eq. (6.1), with explicit values for $L_{10}^{r}(\mu)$ and $C_{87}(\mu)$ which only depend on the resonance masses and the pion decay constant. The logarithmic dependence with the $\chi \mathrm{PT}$ renormalization scale is fully reproduced through the Goldstone loops. The resulting predictions for the two low-energy constants as functions of the $\chi$ PT renormalization scale are shown in Fig. 1 . At the reference scale $\mu_{0}=770 \mathrm{MeV}$, one gets the numerical values [18]:

$$
L_{10}^{r}\left(\mu_{0}\right)=(-4.4 \pm 0.9) \cdot 10^{-3}, \quad C_{87}^{r}\left(\mu_{0}\right)=(3.9 \pm 1.4) \cdot 10^{-3} \mathrm{GeV}^{-2},
$$

where the uncertainties reflect the present errors associated with the input resonance masses. These numbers are in very good agreement with the recent and more precise $O\left(p^{6}\right)$ phenomenolog- 

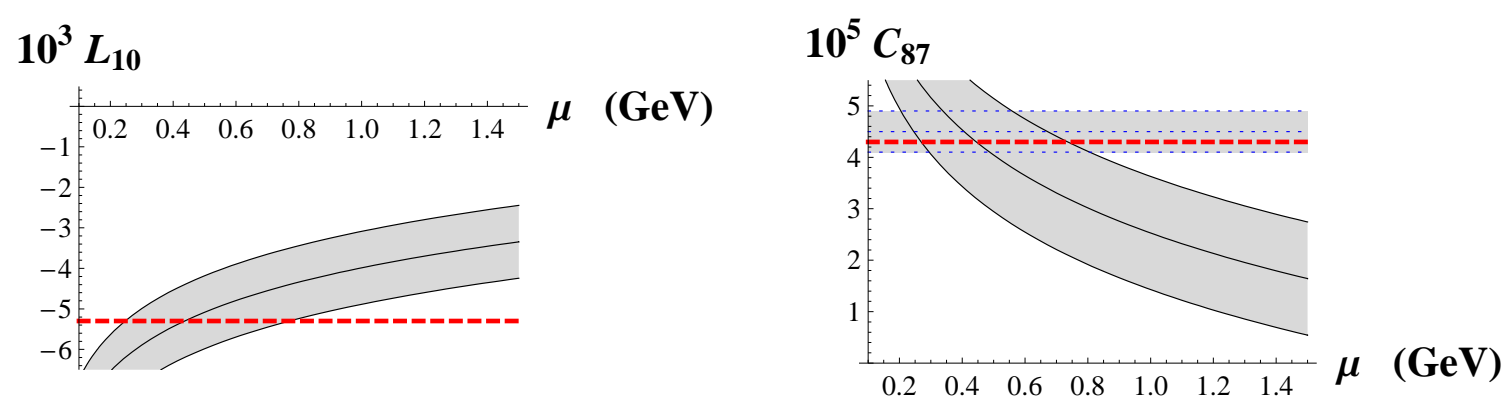

Figure 1: NLO predictions (solid gray bands) for $L_{10}^{r}(\mu)$ (left) and $C_{87}^{r}(\mu)$ (right, $1 / f^{2}$ units), compared to the LO estimates (dashed) and the result from Ref. [36] (dotted) obtained with Pade approximants.

ical determination of these constants from $\tau$-decay data: $L_{10}^{r}\left(\mu_{0}\right)=(-4.06 \pm 0.39) \cdot 10^{-3}$ and $C_{87}^{r}\left(\mu_{0}\right)=(4.89 \pm 0.19) \cdot 10^{-3} \mathrm{GeV}^{-2}[37]$.

The difference between the scalar and pseudoscalar two-point functions, $\Pi_{S-P}(t) \equiv \Pi_{S S}(t)-$ $\Pi_{P P}(t)$, has been also analyzed within $\mathrm{R} \chi \mathrm{T}$, at the NLO, in a completely analogous way [17]. Once more, the short-distance QCD constraints are able to fix all relevant resonance couplings in terms of the pion decay constant and resonance masses. The corresponding low-energy expansion of $\Pi_{S-P}(t)$ provides then a determination of the $\chi \mathrm{PT}$ couplings $L_{8}^{r}(\mu)$ and $C_{38}^{r}(\mu)$ at the NLO in $1 / N_{C}$, keeping full control of the renormalization-scale dependence. At the reference scale $\mu_{0}$, one gets the values [17]:

$$
L_{8}^{r}\left(\mu_{0}\right)=(0.6 \pm 0.4) \cdot 10^{-3}, \quad C_{38}^{r}\left(\mu_{0}\right)=(0.3 \pm 0.8) \cdot 10^{-3} \mathrm{GeV}^{-2} .
$$

The predicted value for $L_{8}$ is in good agreement with the $O\left(p^{6}\right)$ phenomenological determination $L_{8}^{r}\left(\mu_{0}\right)=(0.62 \pm 0.20) \cdot 10^{-3}[38]$.

\section{Summary}

The $1 / N_{C}$ expansion provides a useful bridge between short and long distances and a powerful power-counting parameter. The strong dynamics at $N_{C} \rightarrow \infty$ corresponds to the tree approximation to some local effective Lagrangian (with an infinite number of degrees of freedom). $\mathrm{R} \chi \mathrm{T}$ constitutes an appropriate effective Lagrangian implementation of the large $-N_{C}$ world, incorporating the chiral symmetry constraints. It allows to obtain useful approximations to the QCD Green functions, in terms of a finite number of meson fields, which interpolate between $\chi \mathrm{PT}$ and the underlying QCD theory.

Integrating out the heavy resonance fields one recovers at low energies the $\chi$ PT Lagrangian with explicit values of the chiral couplings in terms of resonance parameters. Since the shortdistance properties of QCD impose stringent constraints on the $\mathrm{R} \chi \mathrm{T}$ couplings, it is then possible to extract information on the low-energy constants of $\chi \mathrm{PT}$.

Truncating the infinite tower of meson resonances to the lowest states with $0^{-+}, 0^{++}, 1^{--}$ and $1^{++}$quantum numbers one gets a very successful prediction of the $O\left(p^{4} N_{C}\right) \chi \mathrm{PT}$ couplings in terms of only three parameters: $M_{V}, M_{S}$ and the pion decay constant $f$. This provides a theoretical 
understanding of the role of resonance saturation in low-energy phenomenology, which has been recently extended to $O\left(p^{6}\right)$.

Hadronic loops generate corrections suppressed by factors of $1 / N_{C}$, which can be analyzed within $\mathrm{R} \chi \mathrm{T}$. The short-distance QCD constraints turn out to be crucial in order to control the ultraviolet behaviour of the effective theory; together with analyticity and unitarity, they allow to determine the Green functions at the NLO in $1 / N_{C}$. Taking the low-energy limit, it is then possible to pin down the $\chi \mathrm{PT}$ couplings at NLO and, therefore, to control their chiral renormalization-scale dependence. Only a few explicit calculations have been done up to now, with very successful results. Further progress is to be expected in the near future [39].

\section{Acknowledgements}

I'm grateful to J. Portolés, I. Rosell and J.J. Sanz-Cillero for useful comments on the manuscript. This work has been supported by MICINN, Spain (grants FPA2007-60323 and Consolider-Ingenio 2010 CSD2007-00042, CPAN), by the EU Contract MRTN-CT-2006-035482 (FLAVIAnet) and by Generalitat Valenciana (PROMETEO/2008/069).

\section{References}

[1] S. Weinberg, Physica A96 (1979) 327.

[2] J. Gasser and H. Leutwyler, Ann. Phys. 158 (1984) 142; Nucl. Phys. B250 (1985) 465, 517, 539.

[3] V. Bernard and U.-G. Meissner, Ann. Rev. Nucl. Part. Sci. 57 (2007) 33; S. Scherer, Adv. Nucl. Phys. 27 (2003) 277; G. Ecker, Prog. Part. Nucl. Phys. 35 (1995) 1; A. Pich, Rept. Prog. Phys. 58 (1995) 563; arXiv:hep-ph/9806303.

[4] J. Bijnens, Prog. Part. Nucl. Phys. 58 (2007) 521; A. Pich, Int. J. Mod. Phys. A20 (2005) 1613.

[5] J. Bijnens, G. Colangelo and G. Ecker, Ann. Phys. 280 (2000) 100; JHEP 9902 (1999) 020; Phys. Lett. B441 (1998) 437.

[6] J. Bijnens, L. Girlanda and P. Talavera, Eur. Phys. J. C23 (2002) 539.

[7] H. W. Fearing and S. Scherer, Phys. Rev. D53 (1996) 315.

[8] G. 't Hooft, Nucl. Phys. B72 (1974) 461; B75 (1974) 461.

[9] E. Witten, Nucl. Phys. B160 (1979) 57.

[10] G. Ecker, J. Gasser, A. Pich and E. de Rafael, Nucl. Phys. B321 (1989) 311.

[11] G. Ecker et al., Phys. Lett. B223 (1989) 425.

[12] V. Cirigliano et al., Nucl. Phys. $\mathbf{B 7 5 3}$ (2006) 139.

[13] A. Pich, arXiv:hep-ph/0205030.

[14] M. Jamin, J.A. Oller and A. Pich, Nucl. Phys. B622 (2002) 279; B587 (2000) 331.

[15] S. Weinberg, Phys. Rev. Lett. 18 (1967) 507.

[16] M. Golterman and S. Peris, Phys. Rev. D61 (2000) 034018.

[17] I. Rosell, J.J. Sanz-Cillero and A. Pich, JHEP 0701 (2007) 039. 
[18] I. Rosell, J.J. Sanz-Cillero and A. Pich, JHEP 0807 (2008) 014.

[19] G. Ecker and C. Zauner, Eur. Phys. J. C52 (2007) 315.

[20] D. Toublan, Phys. Rev. D53 (1996) 6602 [Err: D57 (1998) 4495]; B. Ananthanarayan, Phys. Rev. D58 (1998) 036002.

[21] M. Knecht and E. de Rafael, Phys. Lett. B424 (1998) 335; S. Peris et al., JHEP 9805 (1998) 011; 0201 (2002) 024; Phys. Rev. Lett. 86 (2001) 14; JHEP 0201 (2002) 024.

[22] B. Moussallam, Phys. Rev. D51 (1995) 4939; Nucl. Phys. B504 (1997) 381.

[23] M. Knecht and A. Nyffeler, Eur. Phys. J. C21 (2001) 659.

[24] P.D. Ruiz-Femenía, A. Pich and J. Portolés, JHEP 0307 (2003) 003.

[25] V. Cirigliano, G. Ecker, M. Eidemüller, A. Pich and J. Portolés, Phys. Lett. $B 596$ (2004) 96.

[26] V. Cirigliano, G. Ecker, M. Eidemuller, R. Kaiser, A. Pich and J. Portolés, JHEP 0504 (2005) 006.

[27] J. Bijnens, E. Gámiz, E. Lipartia and J. Prades, JHEP 0304 (2003) 055.

[28] F. Guerrero and A. Pich, Phys. Lett. B412 (1997) 382; D. Gómez Dumm, A. Pich and J. Portolés, Phys. Rev. D62 (2000) 054014; A. Pich and J. Portolés, Phys. Rev. D63 (2001) 093005;

J.J. Sanz-Cillero and A. Pich, Eur. Phys. J. C27 (2003) 587.

[29] E. Pallante and A. Pich, Phys. Rev. Lett. 84 (2000) 2568; Nucl. Phys. $B 592$ (2000) 294; E. Pallante, A. Pich and I. Scimemi, Nucl. Phys. B617 (2001) 441.

[30] T.N. Truong, Phys. Rev. Lett. 61 (1988) 2526; A. Dobado et al., Phys. Lett. $\mathbf{B 2 3 5}$ (1990) 134; T. Hannah, Phys. Rev. D55 (1997) 5613; J.A. Oller and E. Oset, Phys. Rev. D60 (1999) 074023; A. Gómez-Nicola and J.R. Peláez, Phys. Rev. D65 (2002) 054009.

[31] O. Catà and S. Peris, Phys. Rev. D65 (2002) 056014.

[32] I. Rosell, J.J. Sanz-Cillero and A. Pich, JHEP 0408 (2004) 042.

[33] J.J. Sanz-Cillero, Phys. Lett. B649 (2007) 180; L.Y. Xiao and J.J. Sanz-Cillero, Phys. Lett. B659 (2008) 452.

[34] J. Portolés, I. Rosell and, P. Ruiz-Femenía, JHEP 0512 (2005) 020; Phys. Rev. D75 (2007) 114011.

[35] G. Amorós, J. Bijnens and P. Talavera, Nucl. Phys. $\mathbf{B 5 6 8}$ (2000) 319; E. Golowich and J. Kambor, Nucl. Phys. B447 (1995) 373; Phys. Rev. D58 (1998) 036004; S. Durr and J. Kambor, Phys. Rev. D61 (2000) 114025.

[36] P. Masjuan and S. Peris, Phys. Lett. B663 (2008) 61.

[37] M. González-Alonso, A. Pich and J. Prades, Phys. Rev. D in press (arXiv:0810.0760 [hep-ph]).

[38] G. Amorós, J. Bijnens and P. Talavera, Nucl. Phys. B602 (2001) 87.

[39] I. Rosell, J.J. Sanz-Cillero and A. Pich, work in progress. 